\title{
Charged Higgs boson production at the LHC
}

\section{Michael KRÄMER*}

CERN, Geneva, Switzerland and RWTH Aachen University, Aachen, Germany

E-mail: mkraemer@physik.rwth-aachen.de

The dominant production process for heavy charged Higgs bosons at the LHC is the associated production with heavy quarks. We present cross-section predictions for associated heavy-quark Higgs production through the parton processes $q \bar{q}, \mathrm{gg} \rightarrow \mathrm{tbH}^{ \pm}$, including next-to-leading-order supersymmetric QCD corrections. We also present a comparison of the next-to-leading-order results with a calculation based on bottom-gluon fusion $\mathrm{gb} \rightarrow \mathrm{tH}^{ \pm}$.

Prospects for Charged Higgs Discovery at Colliders

27-30 September 2010

Uppsala University, Sweden

* Speaker. 


\section{Introduction}

Many extensions of the Standard Model, in particular supersymmetric theories, require two Higgs doublets leading to five physical scalar Higgs bosons, including two (mass-degenerate) charged particles $\mathrm{H}^{ \pm}$. The discovery of a charged Higgs boson would provide unambiguous evidence for an extended Higgs sector beyond the Standard Model. Searches at LEP have set a limit $M_{\mathrm{H}^{ \pm}}>79.3 \mathrm{GeV}$ on the mass of a charged Higgs boson in a general two-Higgs-doublet model [2]. Within the MSSM, the charged Higgs boson mass is constrained by the pseudoscalar Higgs mass and the $\mathrm{W}$ boson mass through $M_{\mathrm{H}^{ \pm}}^{2}=M_{\mathrm{A}}^{2}+M_{\mathrm{W}}^{2}$ at tree level, with only moderate higher-order corrections [3-6]. A mass limit on the MSSM charged Higgs boson can thus be derived from the limit on the pseudoscalar Higgs boson, $M_{\mathrm{A}}>93.4 \mathrm{GeV}$ [7], resulting in $M_{\mathrm{H}^{ \pm}} \gtrsim 120 \mathrm{GeV}$. At the Tevatron, searches for light charged Higgs bosons in top quark decays $\mathrm{t} \rightarrow \mathrm{bH}^{ \pm}[8,9]$ have placed some constraints on the MSSM parameter space, but do not provide any further generic bounds on $M_{\mathrm{H}}^{ \pm}$.

There are two main mechanisms for charged Higgs boson production at the LHC:

$$
\begin{aligned}
& \text { top quark decay: } \quad \mathrm{t} \rightarrow \mathrm{bH}^{ \pm}+X \quad \text { if } M_{\mathrm{H}^{ \pm}} \lesssim m_{\mathrm{t}}, \\
& \text { associate production: } \mathrm{pp} \rightarrow \mathrm{tbH}^{ \pm}+X \quad \text { if } M_{\mathrm{H}^{ \pm}} \gtrsim m_{\mathrm{t}} .
\end{aligned}
$$

Alternative production mechanisms like quark-antiquark annihilation $q \bar{q}^{\prime} \rightarrow \mathrm{H}^{ \pm}, \mathrm{H}^{ \pm}+$jet production, associated $\mathrm{H}^{ \pm} \mathrm{W}^{\mp}$ production or Higgs pair production have suppressed rates, and it is not yet clear whether a signal could be established in any of those channels (see Ref. [10] and references therein). Some of the above production processes may, however, be enhanced in models with non-minimal flavour violation (see e.g. Ref. [11]).

In this talk we focus on NLO QCD predictions for heavy charged Higgs production through the process $\mathrm{pp} \rightarrow \mathrm{tbH}^{ \pm}+X$.

\section{Heavy charged Higgs production with top and bottom quarks}

Two different formalisms can be employed to calculate the cross section for associated $\mathrm{tbH}^{ \pm}$ production. In the four-flavour scheme (4FS) with no b quarks in the initial state, the lowest-order QCD production processes are gluon-gluon fusion and quark-antiquark annihilation, $\mathrm{gg} \rightarrow \mathrm{tbH}^{ \pm}$ and $q \bar{q} \rightarrow \mathrm{tbH}^{ \pm}$, respectively. Potentially large logarithms $\propto \ln \left(\mu_{\mathrm{F}} / m_{\mathrm{b}}\right)$, which arise from the splitting of incoming gluons into nearly collinear b $\bar{b}$ pairs, can be summed to all orders in perturbation theory by introducing bottom parton densities. This defines the five-flavour scheme (5FS) [12]. The use of bottom distribution functions is based on the approximation that the outgoing $b$ quark is at small transverse momentum and massless, and the virtual $b$ quark is quasi on-shell. In this scheme, the leading-order (LO) process for the inclusive tbH $\mathrm{H}^{ \pm}$cross section is gluon-bottom fusion, $\mathrm{gb} \rightarrow \mathrm{tH}^{ \pm}$. The next-to-leading order (NLO) cross section in the 5FS includes $\mathscr{O}\left(\alpha_{\mathrm{s}}\right)$ corrections to $\mathrm{gb} \rightarrow \mathrm{tH}^{ \pm}$and the tree-level processes $\mathrm{gg} \rightarrow \mathrm{tbH}^{ \pm}$and $q \bar{q} \rightarrow \mathrm{tbH}^{ \pm}$. To all orders in perturbation theory the four- and five-flavour schemes are identical, but the way of ordering the perturbative expansion is different, and the results do not match exactly at finite order. For the inclusive production of neutral Higgs bosons with bottom quarks, $\mathrm{pp} \rightarrow \mathrm{b} \overline{\mathrm{b}} \mathrm{H}+X$, the four- 

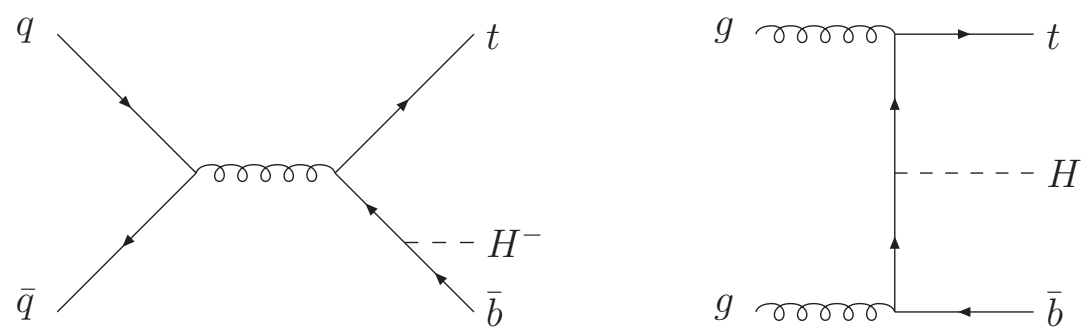

Figure 1: Generic Feynman diagrams for $\mathrm{pp} \rightarrow \mathrm{tbH}^{ \pm}+X$ in the 4FS at the Born level.

and five-flavour scheme calculations numerically agree within their respective uncertainties, once higher-order QCD corrections are taken into account [13-16], see Section 6 of this report.

There has been considerable progress recently in improving the cross section predictions for the associated production of charged Higgs bosons with heavy quarks by calculating NLO SUSYQCD and electroweak corrections in the four and five-flavour schemes [17-25], and the matching of the NLO five-flavour scheme calculation with parton showers [26]. Below, we shall present state-of-the-art NLO QCD predictions in the 4FS, and a first comparison of the 4FS and the 5FS at NLO.

\section{NLO SUSY-QCD predictions for $p p \rightarrow t b H^{ \pm}+X$ in the 4FS}

In the 4FS the production of charged Higgs bosons in association with top and bottom quarks proceeds at $\mathrm{LO}$ through the parton processes $\mathrm{gg} \rightarrow \mathrm{t} \overline{\mathrm{b}} \mathrm{H}^{-}$and $q \bar{q} \rightarrow \mathrm{t} \overline{\mathrm{b}} \mathrm{H}^{-}$, and the charge-conjugate processes with the $\overline{\mathrm{t}} \mathrm{H}^{+}$final state [27-29]. Throughout this talk we present results for the $\overline{\mathrm{t}} \mathrm{H}^{-}$ channels. In NLO QCD the cross section for the charge-conjugate process $\mathrm{pp} \rightarrow \overline{\mathrm{tb}} \mathrm{H}^{+}+X$ at the LHC is identical to $\mathrm{pp} \rightarrow \mathrm{t} \overline{\mathrm{b}} \mathrm{H}^{-}+X$ and can be included by multiplying the results presented below by a factor of two. Generic Feynman diagrams that contribute at LO are displayed in Fig. 1.

The calculation of the NLO QCD corrections to charged Higgs production in the 4FS has been discussed in detail in Ref. [23], both within a two-Higgs-doublet model with the SM particle content besides the extended Higgs sector, and within the MSSM. Below, we present NLO SUSY-QCD predictions for the production of heavy charged MSSM Higgs bosons at the LHC. We consider the SPS $1 b$ benchmark scenario [30] and choose the SM input parameters following the recommendations of the LHC Higgs Cross Section Working Group [31]. The MSTW four-flavour pdf [32] has been adopted.

We first discuss the scale dependence of the total $\mathrm{pp} \rightarrow \mathrm{t} \overline{\mathrm{b}} \mathrm{H}^{-}+X$ cross section at the LHC. The renormalization and factorization scales that enter the hadronic cross section and the running b-quark mass are identified and varied around the central value $\mu_{0}=\left(m_{\mathrm{t}}+m_{\mathrm{b}}+M_{\mathrm{H}^{-}}\right) / 3$, the scale 


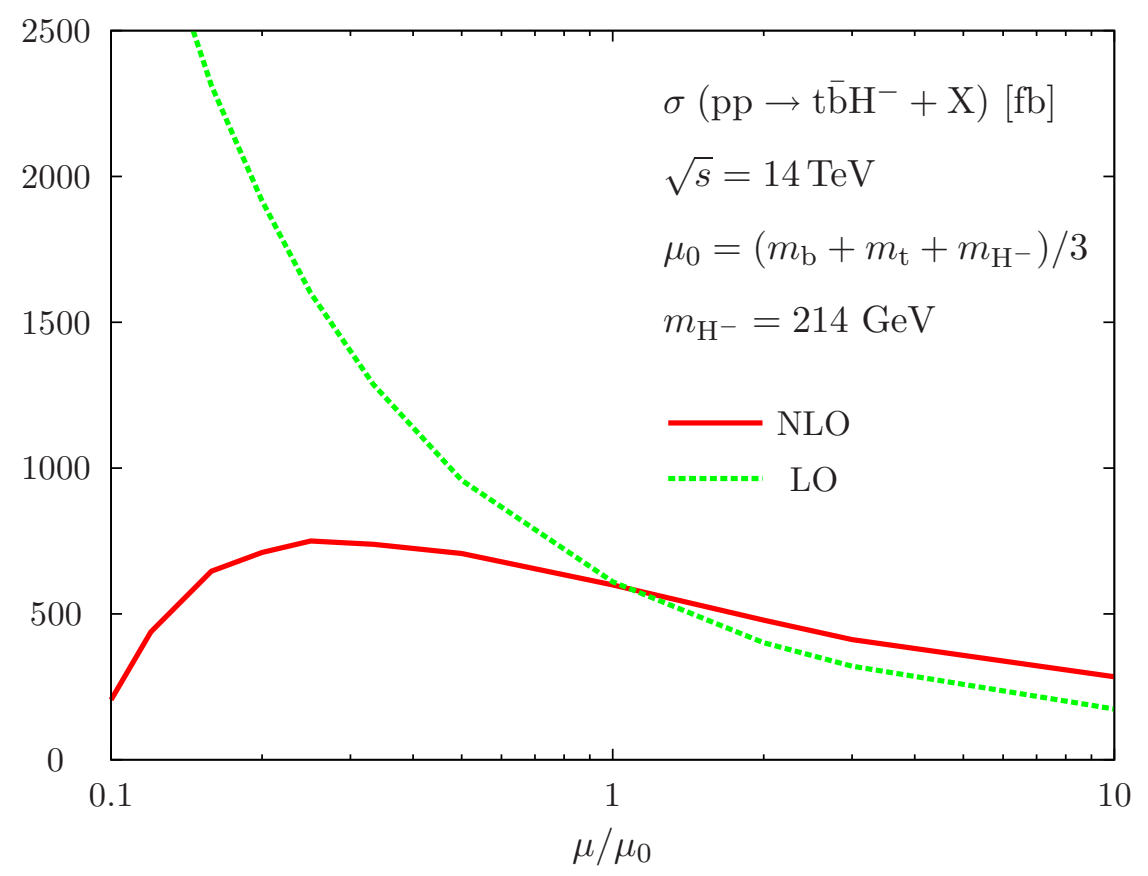

Figure 2: Variation of the LO and NLO cross sections with the renormalization and factorization scales for $\mathrm{pp} \rightarrow \mathrm{t} \overline{\mathrm{b}} \mathrm{H}^{-}+X$ at the LHC.

of $\alpha_{\mathrm{s}}$ in the summation factor of the Yukawa coupling, on the other hand, is kept fixed. Figure 2 shows the scale dependence of the LO and complete NLO SUSY-QCD cross sections at the LHC with $14 \mathrm{TeV}$ energy, for the SPS $1 \mathrm{~b}$ benchmark point and $M_{\mathrm{A}}=200 \mathrm{GeV}$, corresponding to $M_{\mathrm{H}^{ \pm}}=214.27 \mathrm{GeV}$. As anticipated, the scale dependence of the theoretical prediction is significantly reduced at NLO, with a remaining uncertainty of approximately $\pm 25 \%$ when $\mu$ is varied between $\mu_{0} / 3$ and $3 \mu_{0}$, compared to approximately $\pm 100 \%$ at LO. At the central scale, the $\mathrm{K}$ factor, $\mathrm{K}=\sigma_{\mathrm{NLO}} / \sigma_{\mathrm{LO}}$ is close to one. Note, however, that the K-factor strongly depends on the definition of the LO cross section. As described above, our LO cross section prediction includes the summation of a certain class of QCD corrections through a running Yukawa coupling, and has been evaluated using a LO pdf and $\alpha_{\mathrm{s}}$.

The total LO and NLO SUSY-QCD cross sections for $\mathrm{pp} \rightarrow \mathrm{t} \overline{\mathrm{b}} \mathrm{H}^{-}+X$ at the LHC are shown in Figure 3 as a function of the Higgs-boson mass. Note that $t \bar{b} \mathrm{H}^{-}$production at the LHC is dominated by gluon-induced processes which provide more than $95 \%$ of the cross section. The Kfactor is displayed in the lower part of the plot, together with the scale dependence of the LO and NLO predictions. We observe that for our choice of the central scale, $\mu_{0}=\left(m_{\mathrm{t}}+m_{\mathrm{b}}+M_{\mathrm{H}^{-}}\right) / 3$, the $\mathrm{K}$-factor is moderate over the whole range of Higgs-boson masses. Furthermore, the scale dependence is reduced at NLO also for large Higgs masses, indicating that the perturbative expansion is well under control. Representative values for the total cross section at $14 \mathrm{TeV}$ are listed in Table 1 . We note that the MSSM loop-corrections from squark and gluino exchange 


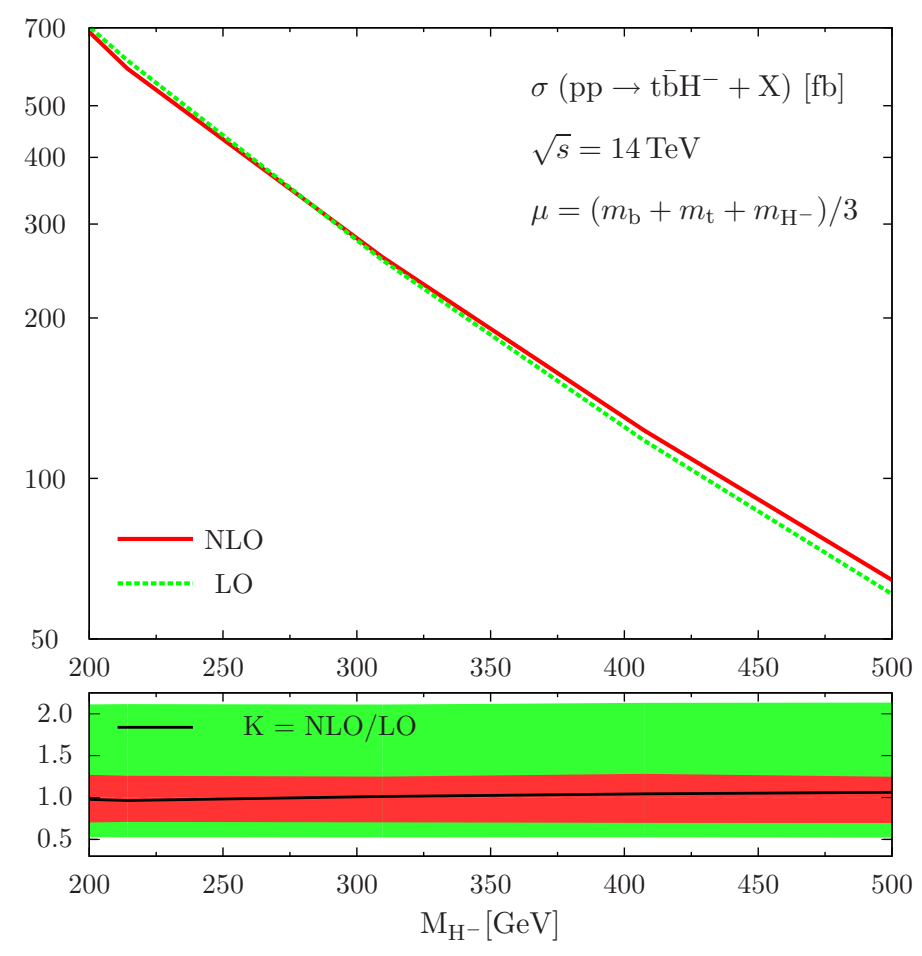

Figure 3: Total LO and NLO cross sections for $\mathrm{pp} \rightarrow \mathrm{t} \overline{\mathrm{b}} \mathrm{H}^{-}+X$ at the LHC as a function of the Higgs-boson mass. The lower plots show the K-factor, $\mathrm{K}=\sigma_{\mathrm{NLO}} / \sigma_{\mathrm{LO}}$, and the scale dependence of the LO and NLO cross section predictions for $\mu_{0} / 3<\mu<3 \mu_{0}$.

are sizable for large $\tan \beta$, but they can be taken into account through the $\Delta_{\mathrm{b}}$-corrections to the bottom-Higgs-Yukawa coupling, i.e. through a rescaling of the NLO-QCD prediction according to $m_{\mathrm{b}} \tan \beta / v \rightarrow m_{\mathrm{b}} \tan \beta / v\left(1-\Delta_{\mathrm{b}} / \tan ^{2} \beta\right) /\left(1+\Delta_{\mathrm{b}}\right)$ (see e.g. [33]).

\section{Comparison with the 5FS calculation}

As discussed in Section 2, in the 5FS the LO process for the inclusive $\mathrm{tH}^{ \pm}$cross section is gluon-bottom fusion, $\mathrm{gb} \rightarrow \mathrm{tH}^{ \pm}$. The NLO cross section includes $\mathscr{O}\left(\alpha_{\mathrm{s}}\right)$ corrections to $\mathrm{gb} \rightarrow \mathrm{tH}^{ \pm}$ and the tree-level processes $\mathrm{gg} \rightarrow \mathrm{tbH}^{ \pm}$and $q \bar{q} \rightarrow \mathrm{tbH}^{ \pm}$, and has been calculated in Refs. [19, 20, 26]. In Figure 4 we present a comparison of the 4FS and 5FS calculations at NLO QCD for the inclusive $\mathrm{pp} \rightarrow \mathrm{tH}^{-}+X$ cross section at the LHC. The 5FS calculation is taken from Ref. [19] and is evaluated with the five-flavour MSTW pdf [34] and the set of input parameters described above. In particular, the renormalization and factorization scales have been set to $\mu_{0}=\left(m_{\mathrm{t}}+m_{\mathrm{b}}+\right.$ $\left.M_{\mathrm{H}^{-}}\right) / 3$, as in the $4 \mathrm{FS}$ calculation. The error band indicates the theoretical uncertainty when the renormalization and factorization scales are varied between $\mu_{0} / 3$ and $3 \mu_{0}$. Thus, the error band also includes the scale choice $\mu_{\mathrm{F}}=\left(m_{\mathrm{t}}+M_{\mathrm{H}^{-}}\right) / 5$ for the 5FS calculation advocated in Refs. [19, 20]. The cross sections shown in Figure 4 do not include the NLO SUSY effects, which can be incorporated within good precision by simply adjusting the bottom Yukawa coupling. Taking the 


\begin{tabular}{|c|c|c|c|c|c|}
\hline \multirow{2}{*}{$M_{\mathrm{A}}[\mathrm{GeV}]$} & \multirow{2}{*}{$M_{\mathrm{H}^{ \pm}}[\mathrm{GeV}]$} & $\bar{m}_{\mathrm{b}}^{\mathrm{NLO}}(\mu)[\mathrm{GeV}]$ & \multicolumn{2}{|c|}{$\sigma\left(\mathrm{pp} \rightarrow \overline{\mathrm{tb}}{ }^{-}+X\right)[\mathrm{fb}]$} & \multirow{2}{*}{$\mathrm{K}=\sigma_{\mathrm{NLO}} / \sigma_{\mathrm{LO}}$} \\
\cline { 4 - 5 } & & & $\mathrm{LO}$ & $\mathrm{NLO}$ & \\
\hline 200 & 214.27 & 2.91 & 609 & $599(2)$ & 0.98 \\
\hline 300 & 309.69 & 2.86 & 257 & $263(1)$ & 1.02 \\
\hline 400 & 407.32 & 2.82 & 118 & $124(1)$ & 1.05 \\
\hline 500 & 505.88 & 2.79 & 58.4 & $62.5(2)$ & 1.07 \\
\hline
\end{tabular}

Table 1: Total cross sections and $\mathrm{K}$-factors for $\mathrm{pp} \rightarrow \mathrm{t} \overline{\mathrm{b}} \mathrm{H}^{-}+X$ at the $\mathrm{LHC}(14 \mathrm{TeV})$. The renormalization and factorization scales are set to $\mu=\left(m_{\mathrm{t}}+m_{\mathrm{b}}+M_{\mathrm{H}^{-}}\right) / 3$. The error from the Monte Carlo integration on the last digit is given in parenthesis if significant. The MSTW four-flavour pdf [32] is adopted. In the third column we show the running b-quark mass evaluated at the default renormalization scale.

scale uncertainty into account, the 4FS and 5FS cross sections at NLO are consistent, even though the predictions in the 5FS at our choice of the central scale are larger than those of the 4FS by approximately 25\%, rather independent of the Higgs-boson mass. Qualitatively similar results have been obtained from a comparison of 4FS and 5FS NLO calculations for single-top production at the LHC [35]. Note that the bottom pdf of the recent five-flavour MSTW fit [34] is considerably smaller than that of previous fits [36] and has lead to a significant decrease in the 5FS cross section prediction.

\section{Acknowledgments}

I would like to thank Stefan Dittmaier, Michael Spira and Manuel Walser for their collaboration. This work is supported in part by the DFG SFB/TR9 "Computational Particle Physics", the Helmholtz Alliance "Physics at the Terascale", and the European Community's Marie-Curie Research Training Network under contract MRTN-CT-2006-035505 "Tools and Precision Calculations for Physics Discoveries at Colliders".

\section{References}

[1] P. W. Higgs, Phys. Lett. 12, 132 (1964); Phys. Rev. Lett. 13, 508 (1964) and Phys. Rev. 145, 1156 (1966); F. Englert and R. Brout, Phys. Rev. Lett. 13, 321 (1964); G. S. Guralnik, C. R. Hagen and T. W. Kibble, Phys. Rev. Lett. 13, 585 (1964).

[2] A. Heister et al. [ALEPH Collaboration], Phys. Lett. B 543 (2002) 1 [arXiv:hep-ex/0207054].

[3] J. F. Gunion and A. Turski, Phys. Rev. D 39 (1989) 2701.

[4] A. Brignole, Phys. Lett. B 277 (1992) 313.

[5] M. A. Diaz and H. E. Haber, Phys. Rev. D 45 (1992) 4246.

[6] M. Frank, T. Hahn, S. Heinemeyer, W. Hollik, H. Rzehak and G. Weiglein, JHEP 0702 (2007) 047 [arXiv:hep-ph/0611326]. 


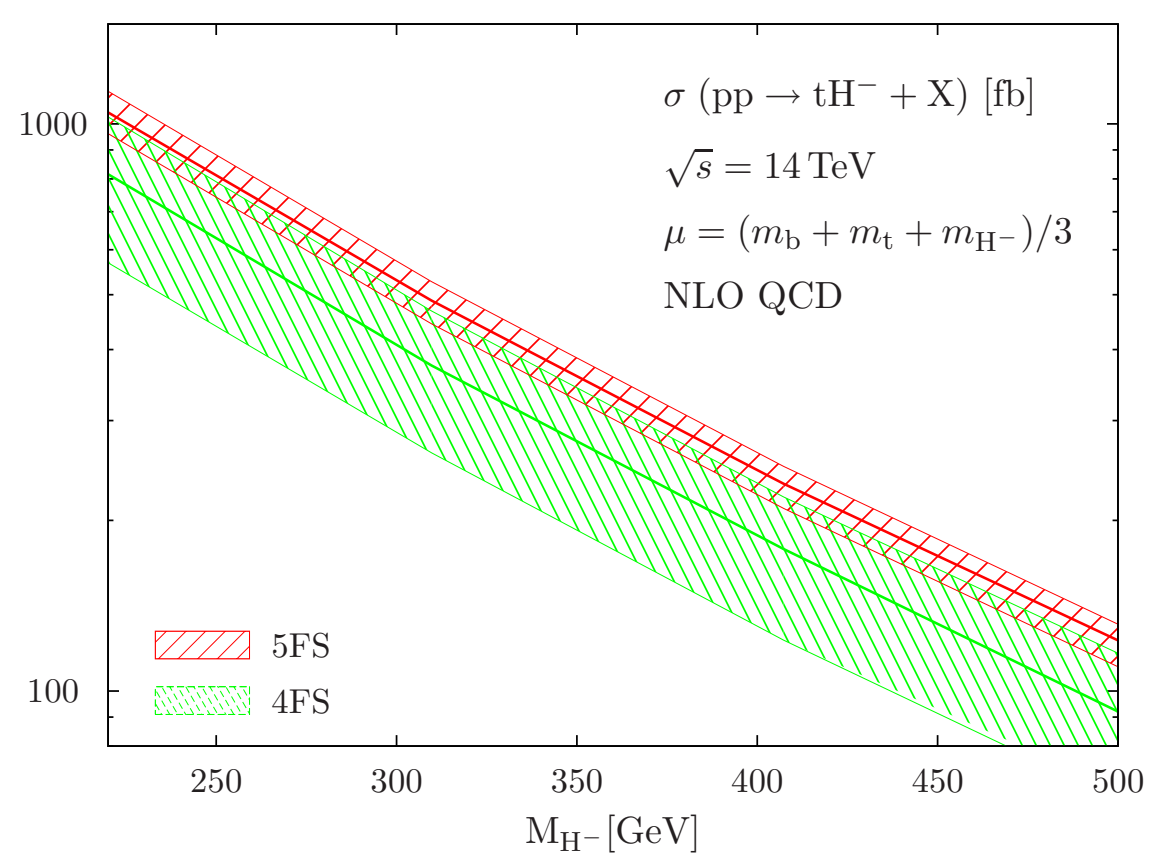

Figure 4: Total NLO cross section for $\mathrm{pp} \rightarrow \mathrm{tH}^{-}+X$ at the LHC as a function of the Higgs-boson mass in the $4 \mathrm{FS}$ and the 5FS. Shown is the central prediction and the scale dependence for $\mu_{0} / 3<\mu<3 \mu_{0}$.

[7] S. Schael et al. [ALEPH Collaboration], Eur. Phys. J. C 47 (2006) 547 [arXiv:hep-ex/0602042].

[8] A. Abulencia et al. [CDF Collaboration], Phys. Rev. Lett. 96 (2006) 042003 [arXiv:hep-ex/0510065].

[9] V. M. Abazov et al. [D0 Collaboration], Phys. Rev. Lett. 88 (2002) 151803 [arXiv:hep-ex/0102039].

[10] A. Djouadi, Phys. Rept. 459 (2008) 1 [arXiv:hep-ph/0503173].

[11] S. Dittmaier, G. Hiller, T. Plehn and M. Spannowsky, Phys. Rev. D 77 (2008) 115001 [arXiv:0708.0940 [hep-ph]].

[12] R. M. Barnett, H. E. Haber and D. E. Soper, Nucl. Phys. B 306 (1988) 697;

D. A. Dicus and S. Willenbrock, Phys. Rev. D 39 (1989) 751.

[13] S. Dittmaier, M. Krämer and M. Spira, Phys. Rev. D 70 (2004) 074010 [hep-ph/0309204].

[14] J. Campbell et al., [hep-ph/0405302].

[15] S. Dawson, C. B. Jackson, L. Reina and D. Wackeroth, Mod. Phys. Lett. A 21 (2006) 89 [hep-ph/0508293]. 
[16] C. Buttar et al., [hep-ph/0604120].

[17] S. h. Zhu, Phys. Rev. D 67 (2003) 075006 [arXiv:hep-ph/0112109].

[18] G. P. Gao, G. R. Lu, Z. H. Xiong and J. M. Yang, Phys. Rev. D 66 (2002) 015007 [arXiv:hep-ph/0202016].

[19] T. Plehn, Phys. Rev. D 67 (2003) 014018 [arXiv:hep-ph/0206121].

[20] E. L. Berger, T. Han, J. Jiang and T. Plehn, Phys. Rev. D 71 (2005) 115012 [arXiv:hep-ph/0312286].

[21] N. Kidonakis, PoS HEP2005 (2006) 336 [arXiv:hep-ph/0511235].

[22] W. Peng, M. Wen-Gan, Z. Ren-You, J. Yi, H. Liang and G. Lei, Phys. Rev. D 73 (2006) 015012 [arXiv:hep-ph/0601069].

[23] S. Dittmaier, M. Kramer, M. Spira and M. Walser, arXiv:0906.2648 [hep-ph].

[24] M. Beccaria, G. Macorini, L. Panizzi, F. M. Renard and C. Verzegnassi, Phys. Rev. D 80 (2009) 053011 [arXiv:0908.1332 [hep-ph]].

[25] N. Kidonakis, arXiv:1005.4451 [hep-ph].

[26] C. Weydert et al., Eur. Phys. J. C 67 (2010) 617 [arXiv:0912.3430 [hep-ph]].

[27] J. L. Diaz-Cruz and O. A. Sampayo, Phys. Rev. D 50 (1994) 6820.

[28] F. Borzumati, J. L. Kneur and N. Polonsky, Phys. Rev. D 60 (1999) 115011 [arXiv:hep-ph/9905443].

[29] D. J. Miller, S. Moretti, D. P. Roy and W. J. Stirling, Phys. Rev. D 61 (2000) 055011 [arXiv:hep-ph/9906230].

[30] B. C. Allanach et al., Eur. Phys. J. C 25 (2002) 113 [eConf C010630 (2001) P125] [hep-ph/0202233].

[31] See https://twiki.cern.ch/twiki/bin/view/LHCPhysics/CrossSections

[32] A. D. Martin, W. J. Stirling, R. S. Thorne and G. Watt, arXiv:1007.2624 [hep-ph].

[33] M. Carena, D. Garcia, U. Nierste and C. E. M. Wagner, Nucl. Phys. B 577 (2000) 88 [hep-ph/9912516].

[34] A. D. Martin, W. J. Stirling, R. S. Thorne and G. Watt, Eur. Phys. J. C 63 (2009) 189 [arXiv:0901.0002 [hep-ph]].

[35] J. M. Campbell, R. Frederix, F. Maltoni and F. Tramontano, Phys. Rev. Lett. 102 (2009) 182003 [arXiv:0903.0005 [hep-ph]] and JHEP 0910 (2009) 042 [arXiv:0907.3933 [hep-ph]].

[36] A. D. Martin, R. G. Roberts, W. J. Stirling and R. S. Thorne, Phys. Lett. B 604 (2004) 61 [arXiv:hep-ph/0410230]. 